\title{
Una aproximación desde la fauna del templete Tulán-54 (Salar de Atacama, Chile) a los nichos en la arquitectura durante inicios del Formativo (ca. 3.300-2.400 AP)
}

\section{The Templete Tulán-54 (Salar de Atacama, Chile) faunas as proxies to interpret architectural niches during the beginning of the Formative period (ca. 3,300-2,400 BP).}

\author{
ISABEL CARTAJENA F. ${ }^{1}$, PATRICIO LÓPEZ M. ${ }^{2}$ \& LAUTARO NÚÑEZ A. ${ }^{3}$ \\ ${ }^{1}$ Departamento de Antropología, Universidad de Chile \\ email: isabel.cartajena@gmail.com \\ ${ }^{2}$ Museo de Historia Natural y Cultural del Desierto de Atacama, Chile \\ email: patriciolopezmend@gmail.com \\ ${ }^{3}$ Instituto de Investigaciones Arqueológicas y Museo (IIAM), \\ Universidad Católica del Norte, Le Paige 380, San Pedro de Atacama, Chile. \\ email: lautaro.nunez@hotmail.com
}

(Received 8 June 2018; Revised 4 September 2018; Accepted 27 October 2018)

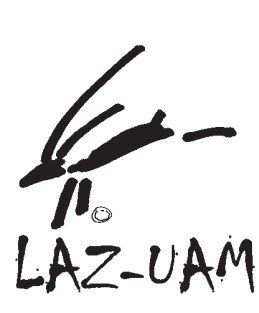

RESUMEN: En el sitio Tulán-54 (3360-2950 a 2410-2370 cal. A.P.) se encuentra una gran estructura semisubterránea, delimitada por un muro perimetral construido a partir de grandes bloques líticos los que fueron dispuestos sobre el piso socavado. Entre los bloques se dejaron cámaras liberadas o nichos entre dos jambas que sostienen dinteles como parte del muro perimetral del templete. En este trabajo presentamos los resultados del análisis zooarqueológico y tafonómico de los restos faunísticos provenientes de los nichos 1, 2, 5 y 6 . Los nichos presentan grandes diferencias en cuanto al número de restos recuperados, siendo el más abundante el Nicho 5. La distribución de unidades anatómicas es similar a la observada en los recintos del templete, al igual que la composición taxonómica. No se observa una selección especial de unidades anatómicas, taxones o grupos etarios, salvo en el Nicho 1 por la presencia de zorro culpeo que en general está bajamente representado en el sitio. En el Nicho 2 se recuperó un espécimen de gran tamaño en el rango de las llamas actuales, el que podría corresponder a un morfotipo carguero. Los restos presentan baja remontabilidad anatómica y mecánica, donde la mayoría son producto del descarte post procesamiento para carne, médula y formatización de artefactos, principalmente de carácter secundario.

PALABRAS CLAVES: FORMATIVO TEMPRANO, CIRCUMPUNA DE ATACAMA, TEMPLETE, NICHOS, FAUNA
ABSTRACT: At the site Tulan-54 (3360-2950 cal. AP to 2410-2370 cal. A.P.), a large sunken ceremonial structure was found. The temple has a surrounding perimeter wall, built with large blocks arranged vertically on the previously dug floor and intercalated by niches composed by lintels and jambs. Zooarchaeological and taphonomic analyses of the animal bones recovered in the Niches 1, 2, 5 and 6 are presented. The number of bones varies between niches being more abundant in niche 5. The distribution and frequency of anatomical units and the taxonomic composition of the niches are very similar to those observed in the inner precincts of the temple 
structure. There is no clear selection of anatomical units, taxonomic or age groups except for few foxes remains recovered in Niche 1, with scarce representation in the bone assemblages recovered in the site. In Niche 2 a large size camelid specimen was recovered in the upper range of modern llamas, suggesting the presence of beast of burdens. Anatomical and mechanical refitting was very low, suggesting the presence of mostly secondary refuses discarded after butchering, marrow consumption and artefact manufacturing.

\section{KEYWORDS: EARLY FORMATIVE, ATACAMA CIRCUMPUNA, TEMPLE STRUCTURE, NICHES, FAUNA}

\section{INTRODUCCIÓN}

Durante el Formativo se observa el desarrollo de arquitectura ceremonial y pública en los Andes centro-sur junto a una serie de artefactos y parafernalia ritual (Stanish, 2003; Bandy, 2006), asociados a prácticas sociales que presentan una amplia distribución en las tierras altas. Los recintos ceremoniales monumentales se han interpretado como lugares de agregación, donde se habrían desarrollado no sólo festines asociados al consumo de alimentos y bebida sino también actividades ceremoniales relacionadas a inhumaciones y a la disposición de ofrendas (Hastorf, 2003, 2008; Beck, 2004). Esta arquitectura andina ceremonial exhibe rasgos comunes como el empotramiento de pilares verticales que sostienen dinteles horizontales, pozos, nichos empotrados a muros, fogones estructurados, recintos pequeños muchas veces circulares y dispuestos en áreas socavadas, entre otros rasgos (Bonnier, 1987; Adán \& Urbina, 2007; Canziani, 2009; Capriles, 2017; Núñez et al., 2017a, 2017b).

En este caso merecen especial referencia los nichos, rasgos arquitectónicos que aparecen en diversos sitios, asumiendo diferentes características, desde hornacinas hasta nichos abiertos y cámaras, siendo más comunes los primeros. No obstante, el conocimiento acerca de los nichos y sus contenidos son escasos para el Formativo. Esta no es la excepción para el sitio Tulán-54 (3450-3080 a 2410-2370 cal. AP), templete ubicado en el borde sureste de la cuenca del Salar de Atacama (Norte de Chile), $3 \mathrm{~km}$ arriba de la quebrada homónima y cercano al nacimiento del arroyo a 2.952 msnm (Ver Figura 1a y 1b). El área que cubre la arquitectura del templete abarca alrededor de $2.800 \mathrm{~m}^{2}$, y su construcción fue secuencial, en donde en primera instancia se socavó y niveló el piso, levantándose el muro perimetral y situando los bloques verticales en el subsuelo esté- ril. Posterior a esto, se levantó el primer nivel con grandes bloques o machones de gran tamaño, dejando nichos entre dos jambas que sostienen dinteles (ver Figura 2a). El carácter ceremonial del sitio se expresa a partir de distintas manifestaciones rituales, entre las que se encuentran 27 inhumaciones de infantes en fosos cercanos al muro perimetral y con fogones estructurados en cubeta y demarcados mediante implementos de molienda fracturados. El templete se levantó entre los 3.050-2.770 a 2.8702.740 años cal. A.P, periodo en el que también se realizaron las inhumaciones de infantes (Núñez et al., 2017a). Posteriormente, el templete comenzó a ser cubierto a través de continuos eventos de descarte, compuestos principalmente por restos osteofaunísticos, líticos, vegetales y cerámica. Una fecha obtenida de los estratos inferiores de uno de los recintos sugiere que este proceso habría comenzado a los 2.720-2.360 cal. A.P. y terminando a los 2.4902.345 cal. A.P (Núñez et al., 2018).

En el presente trabajo abordamos el análisis zooarqueológico y tafonómico de los restos faunísticos registrados en recientes excavaciones en parte de los nichos del sitio Tulán-54. Así, los objetivos del presente trabajo corresponden a: (a) evaluar la composición taxonómica de los restos zooarqueológicos registrados en cada nicho con el fin de evidenciar diferencias y similitudes con el resto de las evidencias del sitio, (b) evaluar la composición etaria de los restos de camélidos de cada nicho y (c) estimar la frecuencia de unidades anatómicas para el caso de los camélidos.

\section{LOS NICHOS DEL TEMPLETE TULÁN: CARACTERÍSTICAS GENERALES}

Los nichos identificados en Tulán-54 se componen de portales con jambas y dinteles empotrados 


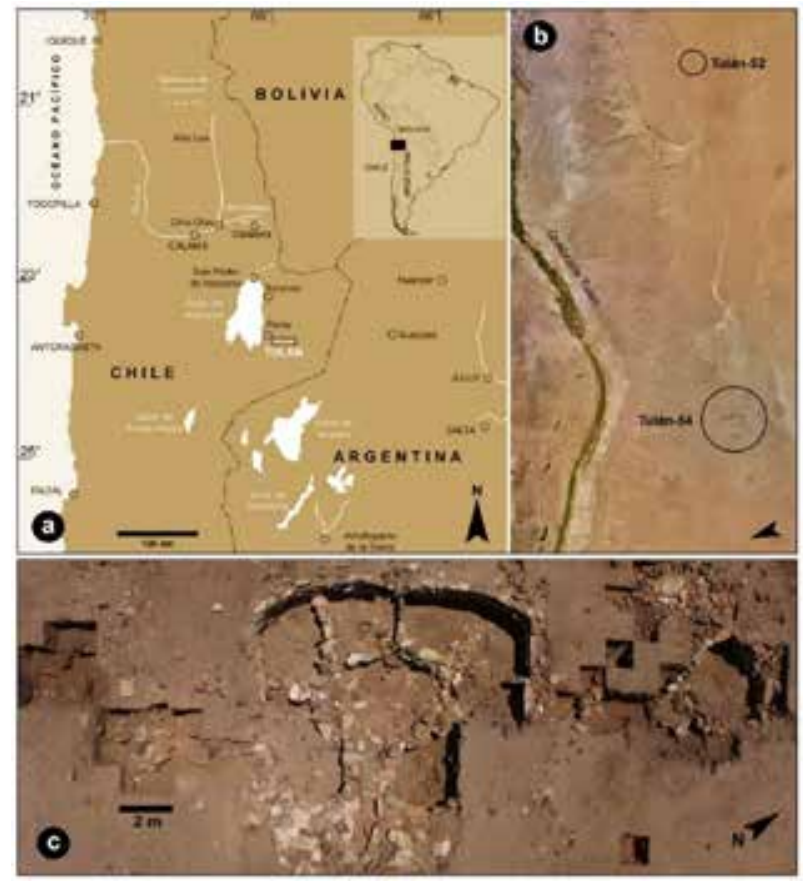

FIGURA 1

(a) Ubicación de la Quebrada de Tulán, (b) vista aérea con la ubicación de los sitios Tulán-52 y Tulán-54, y (c) vista superior del sitio Tu-54 (Imágenes aéreas obtenidas mediante dron por Latorre Geomensura).

a lo largo del muro perimetral (ver Figura 2a y 2b). Las cámaras fueron hechas con lajas horizontales y bloques acumulados, y como rasgos arquitectónicos poseen una raigambre Arcaica Tardía en la quebrada de Tulán (Núñez et al., 2018). Durante la fase Puripica-Tulán, en el sitio Tulán-52 y con fechas que van de los 5.290-4.840 a los 4.430-4.090 cal. AP, dos estructuras subcirculares presentan nichos construidos con lajas verticales. La estructura mayor, evidencia actividades rituales representadas por fosos con ofrendas, un monolito abatido y un nicho conformado por dos jambas y apegado al muro que conecta una pequeña cámara colapsada (Núñez et al., 2018).

En el caso del sitio Tulán-54, un total de 15 nichos han sido contabilizados. Estos nichos presentan dinteles monolíticos bien conservados e incrustados en el piso, y cuyas aperturas se localizan sobre el piso o bien en niveles más altos con bloques en sus bases (Núñez et al., 2018). En la base de cada dintel se dispusieron bloques con caras planas, fragmentos de metates asociados posiblemente a algún tipo de ceremonia. Además, en jambas y dinteles se identificaron en algunos casos, grabados de camélidos e incisiones, uso de pig- mento rojo, restos de fauna en la entrada de cada cámara, y rellenos compuestos por sedimentos finos estériles con restos orgánicos. A decir por las evidencias cerámicas registradas en los nichos y que corresponden al tipo Los Morros, la cronología de los eventos asociados correspondería a la fase Tilocalar, durante el desarrollo del templete Tulán, datada entre los 3.450-3.080 a 2.410-2.370 cal. AP (Núñez et al., 2.018).

El uso recurrente de estos recintos, asociado a la depositación continua de materiales hasta taparlo completamente, trae consigo una serie de problemas en cuanto a la lectura del registro arqueológico. Estos problemas se relacionan a la posibilidad de distinguir en las evidencias artefactuales y ecofactuales ritos particulares o diferencias entre los eventos acaecidos al interior de los recintos en comparación a las actividades desarrolladas afuera. En particular, para el caso de Tulán-54, los procesos de formación son complejos de entender por los ya mencionados eventos cíclicos de sellado intencional del denominado templete (ver Núñez et al., 2017a). Por lo mismo, la excavación de los nichos localizados al interior de esta estructura tuvo por fin el tratar de identificar posibles sectores no 

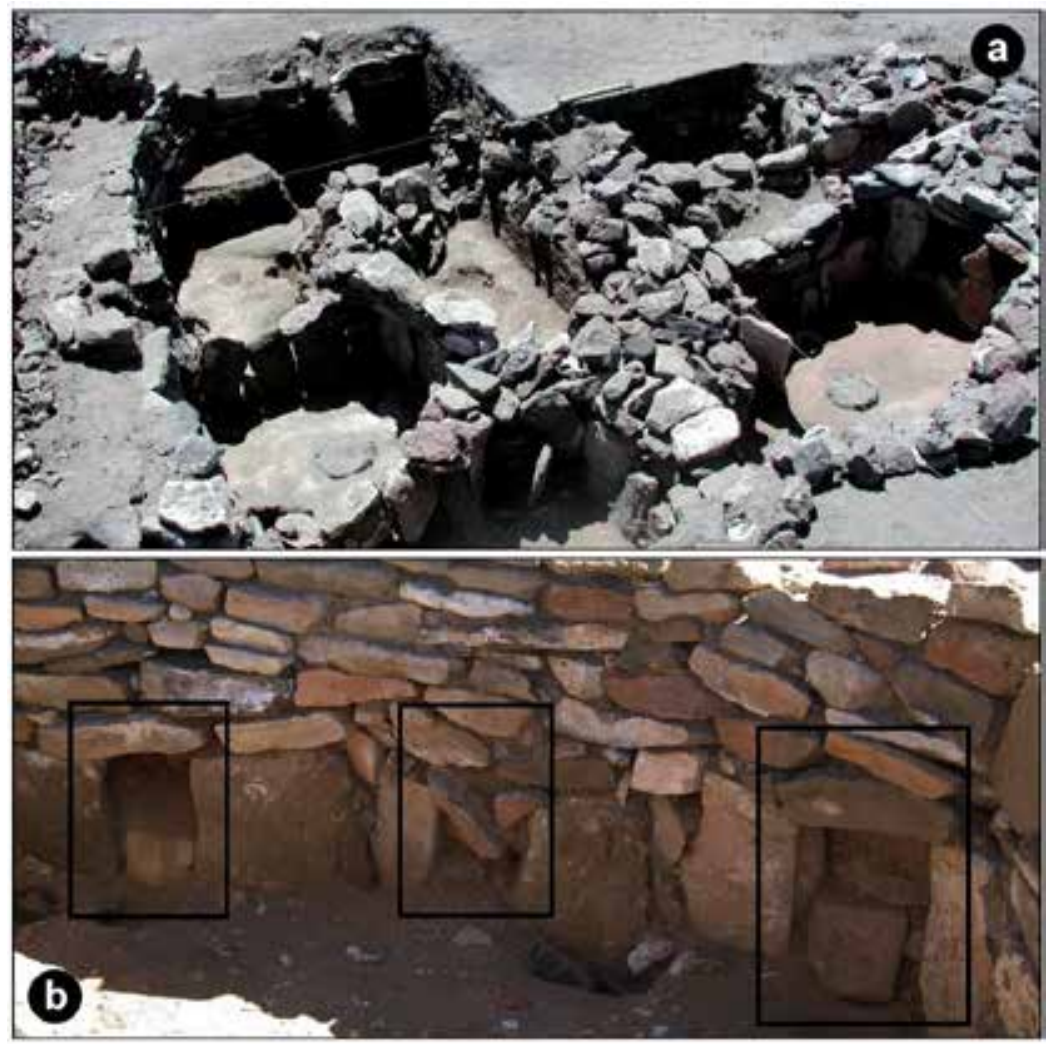

FIGURA 2

(a) Vista general del templete, y (b) vista de nichos empotrados en muro perimetral del templete.

afectos por estas depositaciones cíclicas, sino que más bien, a depósitos previos asociados a actos rituales realizados al interior del templete.

\section{MATERIAL Y MÉTODOS}

Se analizaron los restos arqueofaunísticos asociados a los nichos 1,2, 5 y 6 . El análisis de las evidencias zooarqueológicas fue realizado a partir de las colecciones de referencia del Departamento de Antropología de la Universidad de Chile. Para el caso de los restos de cánidos se contó con la colaboración del Dr. Francisco Prevosti y el Lic. Lucio González con el fin de evaluar los restos de cánidos recobrados. A través del análisis osteométrico de los camélidos se buscó identificar grupos de tamaño que permitiesen distinguir entre las diversas especies silvestres de las domésticas a partir de criterios discutidos en estudios previos (ver Cartajena et al., 2007; Cartajena, 2009). Los estándares métricos utilizados para este análisis corresponden a los propuestos por Driesch (1976), mientras que se evaluó además la exposición a condiciones subaéreas de las muestras mediante la escala de Behrensmeyer (1978) con el fin de entender mejor eventos diferenciales de descarte. La frecuencia de los especímenes óseos se realizó mediante el NISP, MNE, MAU y \%MAU para distinguir tendencias en cuanto a la selección de unidades esqueletales dispuestas en los nichos. Para la determinación de la edad se utilizaron los rangos de Kaufmann (2009) basados en criterios de fusión de las epífisis y las secuencias de brote y desgate dental publicados por Puig (1988).

\section{RESULTADOS}

Se analizaron un total de 797 especímenes provenientes de 4 nichos (Tabla 1). Como se puede observar la mayor cantidad de restos pertenecen a los 
nichos 5 y 6 . Sin embargo, en todos ellos predominan las categorías correspondientes a fragmentos especialmente de huesos largos y astillas, planos y fragmentos mínimos en menor medida (72\%), denotando un alto grado de fragmentación. Los camélidos dominan ampliamente por sobre los otros taxones, sin embargo, la microfauna se encuentra bajamente representada en la mayoría de ellos. En el caso de los redores se identificaron Chinchillidae, Ctenomys sp. y Cricetidae, los que también se encuentran, si bien en bajas proporciones en los rellenos del templete (Labarca, 2005). En el caso de las aves, los restos corresponden a Metriopelia sp. y Passeriformes (Fringillidae) probablemente Silcalis sp. Junto a los restos de aves se encontraron tres fragmentos de cáscaras de huevos de parina en el Nicho 5.

\begin{tabular}{|l|c|c|c|c|c|}
\hline Nicho & Camélidos & Cánidos & Roedores & Aves & NISP \\
\hline Nicho 1 & 17 & 3 & - & - & 20 \\
\hline Nicho 2 & 72 & - & 2 & - & 74 \\
\hline Nicho 5 & 280 & - & 52 & 7 & 339 \\
\hline Nicho 6 & 350 & - & 10 & 4 & 364 \\
\hline Total & 719 & 3 & 64 & 11 & 797 \\
\hline
\end{tabular}

TABLA 1

Distribución taxonómica por nichos (NISP).

Llaman la atención tres especímenes de cánido correspondientes a un radio distal y dos metacarpianos (Mc IV y V) que articulan, lo que sugiere que podrían ser de la misma extremidad anterior. Por tamaño y morfología fueron asignados a $L y$ -

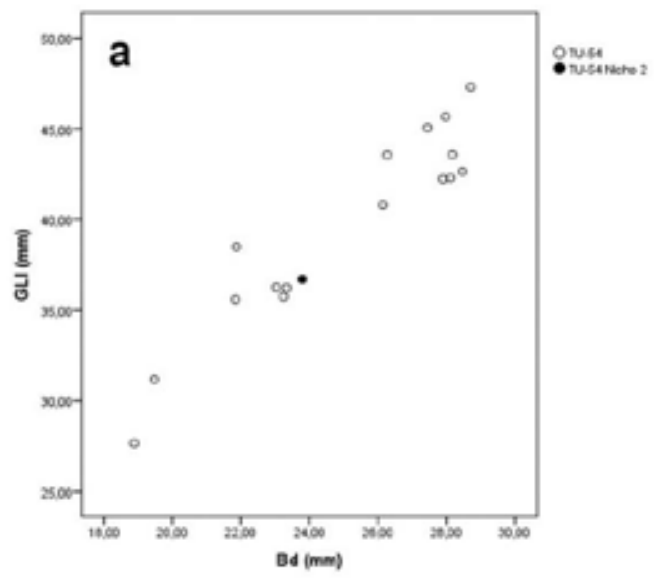

calopex culpaeus (Prevosti y González com. pers). En general los restos de cánidos son muy escasos en el sitio, sólo uno había podido ser también asignado a un zorro (Calas, 2007) y otros tres a Canidae indeterminado (Labarca, 2005).

Si bien gran parte de los especímenes no pudo ser medido, en el caso de los camélidos se observan en todos los nichos ambos grupos de tamaño, el pequeño que corresponde vicuña y el grande a guanaco/llama (Figura 3). En la Figura 3a se detallan las medidas de un astrágalo del Nicho 2 comparado con medidas obtenidas anteriormente de sitio (Cartajena, 2003; Nuñez et al., 2009; Cartajena \& López, 2011), donde se puede observar que pertenece al grupo de tamaño pequeño, el que ha sido interpretado como vicuñas (Cartajena, 2009).

Cinco especímenes, dos primeras falanges, un cuarto carpiano y una escápula corresponden al grupo de camélidos grandes. Llama la atención en el Nicho 2 una escápula de gran tamaño, la que fue comparada con medidas de llamas actuales, algunas de ellas de gran tamaño (Cartajena, 2002), en donde se observa que pertenece a un morfotipo grande, sugiriendo que podría tratarse de una llama posiblemente carguera (Figura 3b).

En todos los nichos, salvo en el 1, predominan las categorías generales. En el Nicho 5 éstas alcanzan el 84\% y en el Nicho 6 el 68\%. Al interior de ellas, los huesos largos y astillas alcanzan el porcentaje más alto. En relación con la representación de unidades anatómicas, en todos los nichos se observa la representación tanto del esqueleto axial

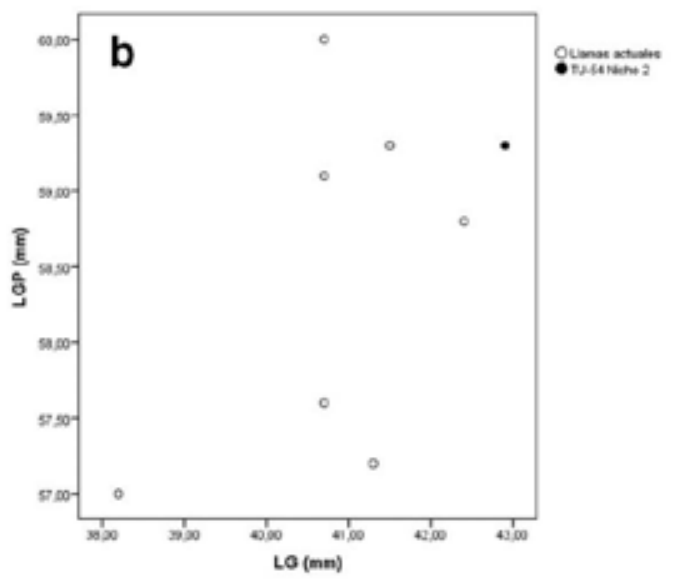

FIGURA 3

(a) Medidas tomadas para el astrágalo asociado al Nicho 2 (GLI y Bd sensu Driesch, 1976) comparadas con medidas anteriores tomadas para TU-54. (b) Medidas tomadas para la escápula asociada al Nicho 2 (LGP y LG sensu Driesch, 1976) comparadas con medidas de llamas actuales (Cartajena, 2002). 
como apendicular. A pesar de las diferencias de tamaño de las muestras, se observa una tendencia similar (Tabla 2).

\begin{tabular}{|l|c|c|c|c|c|}
\hline U. ANATÓMICA & NICHO1 & NICHO 2 & NICHO 5 & NICHO 6 & TOTAL \\
\hline Cráneo & - & - & 6 & 11 & 17 \\
\hline Maxila & 3 & - & - & 3 & 6 \\
\hline $\begin{array}{l}\text { Frag dientes y } \\
\text { molares }\end{array}$ & - & 1 & - & 12 & 13 \\
\hline Mandíbula & 1 & 2 & 1 & - & 4 \\
\hline Axis & - & 1 & - & - & 1 \\
\hline V. cervical & 1 & 1 & 5 & 1 & 8 \\
\hline V. torácica & - & - & 1 & 3 & 4 \\
\hline V. lumbar & - & - & 3 & 4 & 7 \\
\hline Costillas & - & 3 & 6 & 14 & 23 \\
\hline Escápula & - & 3 & 3 & 3 & 9 \\
\hline Húmero diaf. & 1 & 5 & 4 & 1 & 11 \\
\hline Radioulna px & - & - & 1 & - & 1 \\
\hline Radioulna diaf & - & - & - & 1 & 1 \\
\hline Radioulna ds & 1 & 1 & - & - & 2 \\
\hline Carpiano & 2 & 1 & 2 & 3 & 8 \\
\hline Pelvis & 1 & 1 & 1 & - & 3 \\
\hline Fémur px & - & - & 1 & - & 1 \\
\hline Fémur diaf & - & - & 1 & 2 & 3 \\
\hline Astrágalo & - & 1 & - & 2 & 3 \\
\hline Calcáneo & - & - & 1 & 1 & 2 \\
\hline Metatarso px & 1 & 2 & - & - & 3 \\
\hline Metapodios px & - & - & - & 1 & 1 \\
\hline Metapodios diaf & - & 3 & 5 & 11 & 19 \\
\hline Metapodios ds & - & 7 & 3 & 2 & 12 \\
\hline 1ra Fa & 3 & 3 & 12 & 12 & 30 \\
\hline 2da Fa & 1 & 1 & 3 & 5 & 10 \\
\hline 3ra Fa & - & - & 1 & - & 1 \\
\hline H. cortos & - & - & - & 2 & 2 \\
\hline H. largos & 1 & 23 & 40 & 167 & 231 \\
\hline H. planos & 1 & 6 & 32 & 8 & 47 \\
\hline Astillas & & 7 & 42 & 53 & 102 \\
\hline F. mínimos & & - & 166 & 28 & 194 \\
\hline Total & $\mathbf{7 2}$ & $\mathbf{2 8 0}$ & $\mathbf{3 5 0}$ & $\mathbf{7 1 9}$ \\
\hline
\end{tabular}

TABLA 2

Frecuencia unidades anatómicas de camélidos (NISP).

Se graficó para el nicho 5 la frecuencia de unidades anatómicas mínimas en términos de \%MAU. Un alto número de unidades y porciones de otras unidades se encuentran ausentes. Se puede observar que el cráneo, mandíbula y vértebras cervicales se encuentran bien representados a diferencia del restante segmento vertebral y costillas escasamente registradas. Por su parte, el miembro anterior y posterior superior y medio alcanzan una representación media a excepción de la cintura escapular.
La extremidad inferior representada por metapodios y falanges alcanza una mayor representación en relación con las extremidades anteriores (Figura 4). En cuanto a la estructura etaria, se observa una alta representación del conjunto de animales jóvenes (no fusionados) en la mayoría de los nichos, alcanzando el 54\% (en términos de MNE) en el Nicho 2 , donde además se registró un perinato, probablemente nonato (feto). Los rangos etarios para los conjuntos de jóvenes de los nichos 5 (46\% en términos de MNE) y 6 ( $34 \%$ en términos de MNE) muestran gran similitud (Tablas 3 y 4). La mayoría de los especímenes son menores de 24 meses, no obstante, se encuentran algunos neonatos en muy baja proporción. En el caso del Nicho 2, se registró un perinato, probablemente nonato (feto). Además, en el Nicho 1 se identificó una hemimandíbula con la serie dental completa $\left(\mathrm{I}_{3}, \mathrm{C}_{1}, \mathrm{P}_{3}, \mathrm{P}_{4}\right.$, $\mathrm{M}_{1}, \mathrm{M}_{2} \mathrm{y} \mathrm{M}_{3}$ ), perteneciente a una hembra de 3 a 4 años (Puig, 1988). Lo anterior muestra una amplia representación de rangos etarios, sin embargo, la presencia de neonatos sugiere al menos ocupaciones estivales.

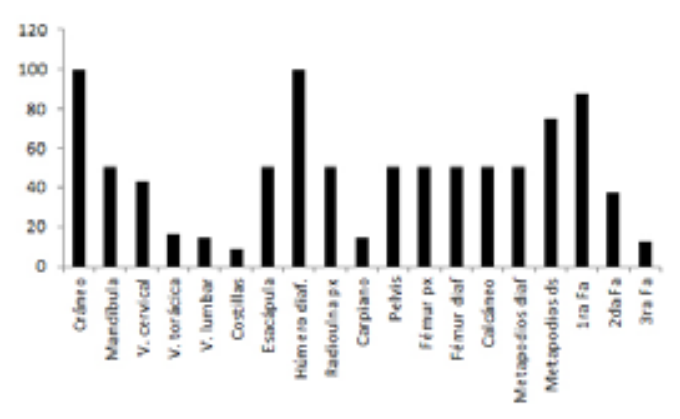

FIGURA 4

Representación de unidades anatómicas (\%MAU) del Nicho 5.

\begin{tabular}{|l|c|c|c|}
\hline Unidad anatómica & NISP & MNE & Rango \\
\hline V. lumbar & 1 & 1 & Menor 36 meses \\
\hline Fémur (cabeza) & 1 & 1 & Menor 30 meses \\
\hline Metapodio & 5 & 3 & Menor 30 meses \\
\hline Costilla & 3 & 3 & Menor 24 meses \\
\hline 1ra Fa px & 3 & 3 & Menor 19 meses \\
\hline 2da Fa px & 1 & 1 & Menor 19 meses \\
\hline Escápula & 1 & 1 & Menor 3 meses \\
\hline Ulna & 1 & 1 & Menor 3 meses \\
\hline Órbita & 1 & 1 & - \\
\hline Total & $\mathbf{1 7}$ & $\mathbf{1 5}$ & \\
\hline
\end{tabular}

TABLA 3

Conjunto óseo no fusionado Nicho 5. 


\begin{tabular}{|l|c|c|c|}
\hline Unidad anatómica & NISP & MNE & Rango \\
\hline V. lumbar & 1 & 1 & Menor 36 meses \\
\hline Costillas & 5 & 5 & Menor 24 meses \\
\hline 1ra Fa px & 3 & 3 & Menor 19 meses \\
\hline 2da Fa px & 2 & 2 & Menor 19 meses \\
\hline Cráneo (Vómer) & 1 & 1 & Menor 12 meses \\
\hline Metapodio & 1 & 1 & Menor 3 meses \\
\hline Total & $\mathbf{1 3}$ & $\mathbf{1 3}$ & \\
\hline
\end{tabular}

TABLA 4

Conjunto óseo no fusionado Nicho 6 .

El estado de conservación es bueno. Tal como se puede observar en la Figura 5, en todos los nichos dominan los estadios 0 y 1 , sugiriendo una rápida depositación de los restos, evitando la exposición más prolongada a condiciones subaéreas. En el caso de los nichos 1 y 2 se observan más grados de meteorización, aunque escasamente representados, lo que sugiere diversos eventos de acumulación o diferentes trayectorias de los restos dispuestos en los nichos. La baja meteorización se explica también por su ubicación al interior de los nichos donde quedaron resguardados de la exposición.

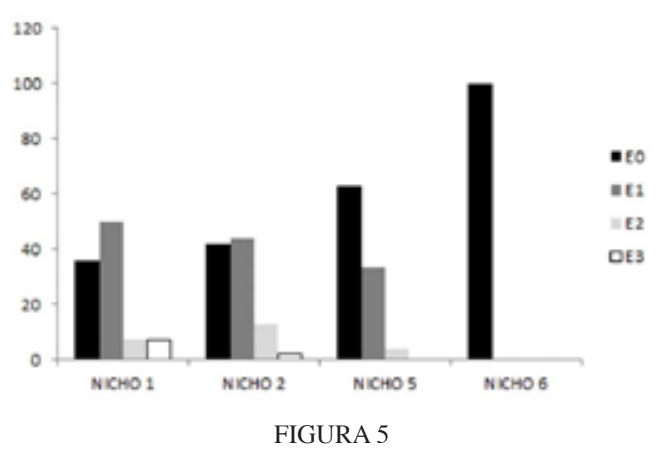

Estado de conservación de los restos de acuerdo con los estadios de meteorización de Behrensmeyer (1978).

Las huellas indican que los restos son producto del procesamiento, ya sea descarne, fracturas y posibles etapas iniciales de formatización de artefactos. Las modificaciones más frecuentes producto de la acción antrópica son las huellas de corte en 9 especímenes (diáfisis de radioulna, pelvis, costilla, vértebra lumbar y una diáfisis de húmero, fémur y tres en fragmentos de diáfisis). Por otra parte, se registraron huellas de impacto asociadas a percusión en cuatro especímenes (radioulna, metapodios y húmero) relacionadas posiblemente con la obtención de médula o para generar matrices para artefactos. Un fragmento de metapodio y un hueso largo presentan una serie de golpes en el borde y otros dos evidencian el desprendimiento de lascas óseas. Finalmente, una primera falange se encuentra modificada, ya que se removieron intencionalmente los cóndilos distales. Solo se registraron 8 fragmentos expuestos a la acción térmica en diversos grados (golpes de fuego y carbonizados), uno de ellos corresponde a un fragmento de hueso largo y los restantes a fragmentos mínimos.

\section{DISCUSIÓN Y CONCLUSIONES}

Los resultados del análisis sugieren que los restos registrados en los nichos pertenecerían a distintos eventos depositacionales, denotados por la diversidad taxonómica (camélidos del grupo de tamaño grande y pequeño), diversos rangos etarios, baja remontabilidad (anatómica y mecánica) y en algunos casos diversos estadios de meteorización. La mayoría de los restos son producto del descarte post procesamiento para carne, médula y formatización de artefactos. Con el fin de comparar los resultados obtenidos del análisis de los nichos, estos fueron contrastados con resultados del análisis de los rellenos de algunas unidades al interior de templete, las cuales corresponden a las cuadrículas $\mathrm{H} 4, \mathrm{H} 2$ y C7 ubicadas cada una en recintos interiores $(\mathrm{C}, \mathrm{B}, \mathrm{E})$. Se consideró la primera unidad estratigráfica denominada Zona Estratigráfica Temprana (ZET), la que está asociada al comienzo de los rellenos en todos los recintos que cubren el piso original y los rasgos como entierros, fogones y pozos entre otros. Al igual que lo observado en los nichos, los restos se encuentran mayoritariamente compuestos por camélidos, y marginalmente a roedores como Chinchillidae, Ctenomys sp. y Cricetidae, junto a aves como paseriformes, Metriopelia sp, y Phoenicopteridae (Labarca 2005; Calas, 2007). En cuanto a los camélidos, el análisis osteométrico sugiere la presencia de Lama guanicoe, Vicugna vicugna y Lama glama (Núñez et al., 2017a; Cartajena et al., 2018).

Si bien el número total de restos es considerablemente mayor, la representación de unidades anatómicas presenta una tendencia similar a lo registrado en los nichos, sin embargo, el cráneo tiene una menor representación, al igual que los miembros superiores delanteros y traseros (húmero y fé- 
H4 \% MAUZET
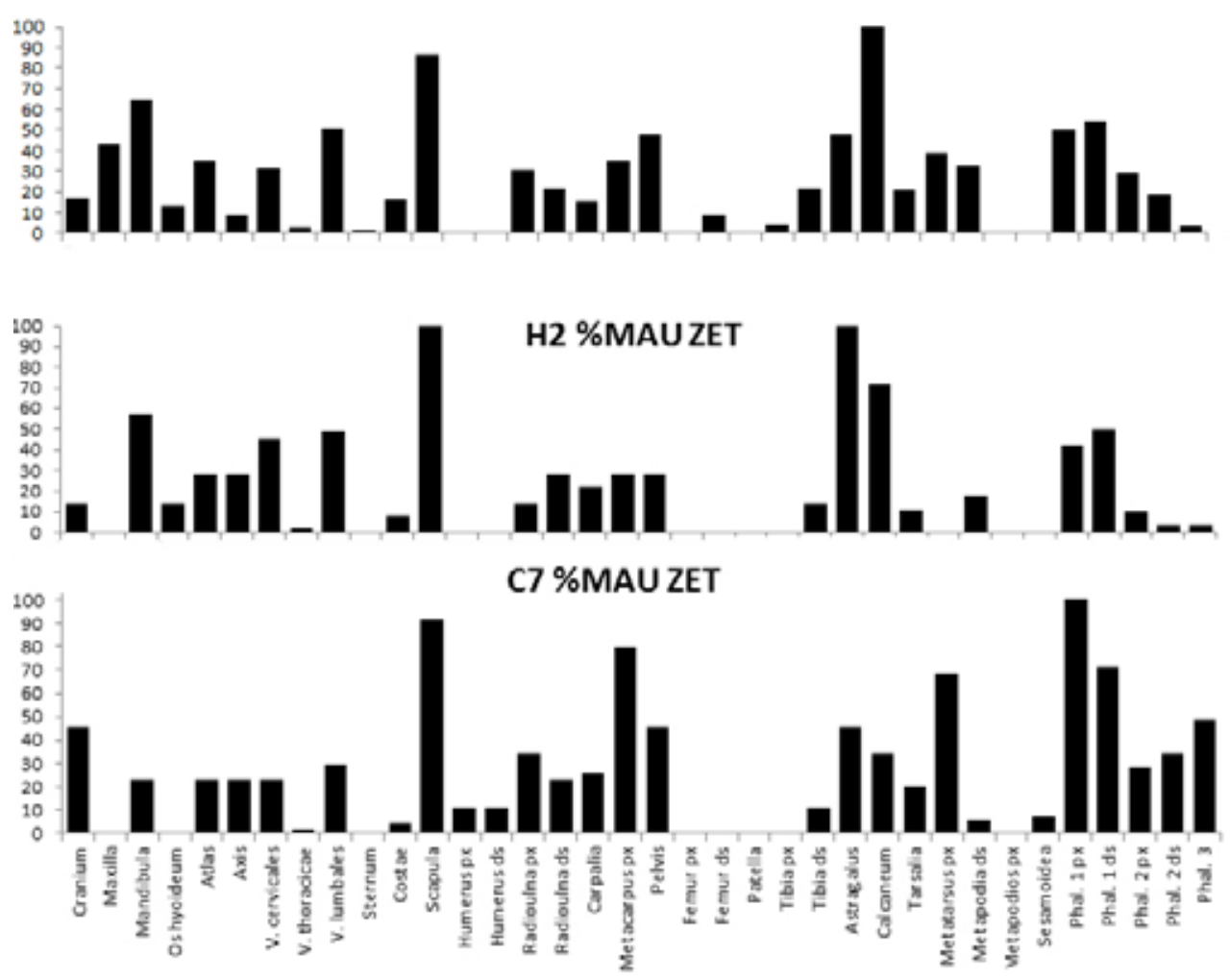

FIGURA 6

Distribución de unidades anatómicas en la Zona Estratigráfica Temprana (ZET) en tres cuadrículas interiores, correspondientes a los recintos C, B y E respectivamente. Los datos para la cuadrícula C7 fueron modificados de Calas (2007). Fuente Cartajena et al. (2018: figura 7).

mur) que están escasamente representados (Figura 6). Los restos al interior de las estructuras muestran en contraposición una alta frecuencia de huesos cortos. Lo anterior permite señalar que no existe una fuerte diferencia en cuanto a las unidades anatómicas registradas tanto en los nichos como en los rellenos del templete en diferentes recintos. Si bien existen diferencias en las frecuencias, no se observa una selección clara de unidades anatómicas en los nichos.

Por su parte, el perfil etario al interior del templete indica la selección de individuos subadultos con un 35\% (\% MNE) y una gran mayoría de animales que van entre los 2 años a 9 meses y con un porcentaje constante que oscila entre el 10 al $20 \%$ de neonatos entre el conjunto de camélidos subadultos (Núñez et al., 2017b; Cartajena et al., 2018). Al comparar la estructura etaria con los resultados de los nichos se observa un comportamiento similar. El conjunto de no fusionados también alcanza valores altos, predominando los especímenes menores de dos años con un porcentaje bajo de neonatos. Finalmente, al comparar los estadios de meteorización, estos siguen la misma tendencia que el resto del sitio, mostrando una rápida depositación. No obstante, en el caso de los nichos, su ubicación los protegió de la exposición a condiciones subaéreas.

En un estudio reciente acerca de los patrones de descarte observados en el sitio Tulán-54 y la colmatación de la estructura ceremonial, es posible identificar tanto un patrón temporal como espacial recurrente al interior como al exterior del templete. Los restos al interior del templete corresponderían mayoritariamente a desechos secundarios derivados del procesamiento, consumo y festines, 
actividades que generaron basuras al exterior del templete y luego fueron ritualmente incorporadas al interior (Cartajena et al., 2018).

En este contexto, los restos al interior de los nichos no guardan relación con el contenido original de estos, el que debió estar relacionado con las actividades rituales en torno al entierro de neonatos y estructuras de combustión. Al igual que lo propuesto por otros autores, pensamos que estas estructuras y entierros originalmente se asocian a parafernalia ritual relacionada con el acto de ofrendar en los nichos. Si bien nuestro conocimiento es parcial ya que solo se excavó la entrada de los nichos, se postula que el material recuperado correspondería a la etapa en que se empieza a rellenar el templete, lo que significó la colmatación no sólo de las estructuras sino también de los nichos. En este contexto, no es posible delimitar contextos primarios o de actividades in situ. No se observa una selección especial de unidades anatómicas, taxones o grupos etarios en el caso de los camélidos, salvo en el Nicho 1 donde se encuentra parte de lo que podría ser la extremidad anterior de un mismo individuo identificado como de zorro culpeo. Si bien los restos de este último no presentan modificaciones, su ubicación al interior del nicho podría ser un remanente de la disposición intencional pero no es posible asociarlos por el momento a alguna actividad ritual particular. Por su parte, los restos de roedores y aves se encuentran bajamente representados en los nichos como en el sitio. Sin embargo, el estudio del contenido de dos fogones dispuestos el piso original sugiere la presencia de aves y roedores, donde si bien los restos de camélidos son también los más abundantes, no podemos descartar su rol en actividades ceremoniales (Cartajena \& Labarca, 2006).

Los rellenos se habrían generado en el contexto de actividades recurrentes de agregación en los cuales se fueron generando los desechos que fueron descartados en el templete y posiblemente en los nichos, lo que puede ser considerado como un comportamiento ritual reiterado a través del tiempo, como lo denota la sucesión estratigráfica de eventos (Núñez et al., 2017a, 2017b). Estos podrían estar asociados con las deidades, antepasados, wakas y elementos de la naturaleza a los que se les brinda sacrificios y comidas (Arnold, 1992; Tomoeda, 1993; Fernández Juárez, 1994; Bolin, 1998). Los pastores altiplánicos organizan encuentros durante periodos específicos del año para que deidades como las Conopas no sientan hambre por lo que los pagos mantienen una serie de elementos de reciprocidad (ver Núñez et al., 2018). Por otra parte, es necesario considerar que, durante el Formativo Temprano la agregación y celebración de festines en los Andes centro-sur adquirieron especial relevancia ya sea como una forma de resolver disputas, reforzar lazos de parentesco e identidades locales (Ikehara \& Shibata, 2005; Hastorf, 2008).

\section{AGRADECIMIENTOS}

Este trabajo se desarrolló en el marco del Proyecto FONDECYT 1130917. Los autores agradecen el permanente apoyo recibido de la Comunidad Atacameña de Peine y a todos los integrantes del equipo arqueológico de Tulán.

\section{REFERENCIAS}

Adán, L. \& Urbina, S. 2007: Arquitectura formativa en San Pedro de Atacama. Estudios Atacameños 34: 7-30.

ARnOLD, D. 1992: The house of earth-bricks and Inka stones: gender, memory and cosmos in Qaqachaka. Journal of Latin American Lore 17(1): 3-69.

BANDY, M. 2006: Early village society in the Formative period in the southern Lake Titicaca Basin. In: Isbell, W. \& Silverman, H. (eds.): Andean Archaeology III. North and South: 210-236. Springer, New York.

Beck Jr, R.A. 2004: Architecture and Polity in the Formative Lake Titicaca Basin, Bolivia. Latin American Antiquity 15(3): 323-343.

Behrensmeyer, A.K. 1978: Taphonomic and ecologic information from bone weathering. Paleobiology 4: 150-162.

Bolin, I. 1998: Rituals of respect: The secret of survival in the high Peruvian Andes. University of Texas Press, Austin.

BonNiER, E. 1987: Les architectures précéramiques dans la Cordillère des Andes. Piruru face à la diversité des donées. L'Anthropologie 92(4): 875-890.

Calas, E. 2007: Análisis de un conjunto arqueofaunístico formativo temprano: Templete Tulán-54, estructura E. Práctica Profesional Inédita, Departamento de Antropología, Universidad de Chile.

Canziani, J. 2009: Ciudad y territorio en los Andes. Contribución a la historia del urbanismo prehispánico. Fondo Editorial, Pontificia Universidad Católica del Perú, Lima. 
CAPRILES, J. 2017: Arqueología del pastoralismo temprano en camélidos en el Altiplano central de Bolivia. Colección Travaux de I'nstitut Francais d'Etudes Andines, Instituto Francés de Estudios Andinos, Plural Editores, La Paz.

CARTAJEnA, I. 2002: Los conjuntos arqueofaunísticos del Arcaico Temprano en la Puna de Atacama, Norte de Chile. Tesis Doctoral Inédita. Freie Universität Berlin.

- 2003: Análisis de las colecciones arqueofaunísticas de los sitios Tulán 52 (TU-52), Tulán-54 (TU-54), Tulán-55 (TU-55), Tulán-57 (TU-57), Tulán-67 (TU67), Tulán-85 (TU-85). (Informe de Avance) Proyecto FONDECYT 1020316. Informe inédito.

- 2009: Explorando la variabilidad morfométrica del conjunto de camélidos pequeños durante el Arcaico Tardío y el Formativo Temprano en quebrada Tulán, Norte de Chile. Revista del Museo de Antropología 2: 199-212.

Cartajena, I. \& Labarca, R. 2006: Small mammals in the Salt Puna: rodent utilization from the Early Archaic to the Early Formative (11.000-2.500 BP), Northern Chile. Ponencia presentada International Council for Archaeozoology, $10^{\text {th }}$ Conference, Abstracts: 44-45.

Cartajena, I. \& López, P. 2011: Análisis de los restos arqueofaunísticos del sitio TU-54: cuadrícula 12 NW, 1W, 1X, 1Y Y 2X NW. (Informe Técnico Nº4). Avance proyecto FONDECYT 1070040. Informe inédito.

Cartajena, I.; Núñez, L. \& Grosjean, M. 2007: Camelid domestication in the western slope of the Puna de Atacama, Northern Chile. Anthropozoologica 42(2): 155-173.

Cartajena, I.; López, P.; Santander, B. \& Núñez, L. 2018: The nature of discarding practices among early herders in the western slope of the Puna de Atacama, Chile (ca. 3300-2300 years cal B.P.). In: International Symposyum 2016. Animals Cultural Identifiers in Ancient Societies? Carl Friedrich von Siemens Stiffung, Munich. En prensa.

DRIESCH, A. von den 1976: A guide to measurement of animal bones from archaeological sites. Peabody Museum Bulletins 1, Harvard University, Cambridge.

FERNÁNDEZ JuÁREZ, G. 1994: El banquete aymara: aspectos simbólicos de las mesas rituales aymaras. Revista Andina 12(1): 155-190.
HASTORF, C. 2003: Community with the ancestors: ceremonies and social memory in the Middle Formative at Chiripa, Bolivia. Journal of Anthropological Archaeology 22(4): 305-332.

- 2008: The Formative period in the Titicaca basin. In: Silverman, H. \& Isbell, W. (eds.): Handbook of South American Archaeology: 545-561. Springer, New York.

Ikebara, H. \& Shibata, K. 2005: Festines e integración social en el periodo formativo: nuevas evidencias de Cerro Blanco, valle bajo de Nepeña. Boletín de Arqueología PUCP 9: 123-159.

KaUfMANN, C. 2009: Estructura de edad y sexo en guanaco. Estudios actualísticos y arqueológicos en Pampa y Patagonia. Sociedad Argentina de Antropología, Buenos Aires.

LABARCA, R. 2005: Zooarqueología de fauna menor en la Puna Salada: El caso de Quebrada Tulán (II Región, Chile). Tesis de Licenciatura Inédita, Departamento de Antropología, Universidad de Chile.

Núñez, L.; Cartajena, I.; De Souza, P. \& Carrasco, C. 2009: Temprana arquitectura ceremonial en la Puna de Atacama (Norte de Chile). Andes 7: 459-490.

Núñez, L.; Cartajena, I.; Carrasco, C.; López, P.; Rivera, F.; De Souza, P.; Santander, B. \& Loyola, R. 2017a: Presencia de un centro ceremonial Formativo en la Circumpuna de Atacama. Chungara 49(1): 3-33.

- 2017b: The temple of TU-54: New insights concerning ceremonial architecture of the Early Formative in the Atacama desert. Antiquity 91(358): 901-915.

Núñez, L.; Cartajena, I.; López, P.; Carrasco, C.; Valenzuela, M. \& Bravo, A. 2018: Nichos, cámaras y ceremonias en el Templete Tulán54 (Circumpuna de Atacama, Chile). Bulletin de Institut Français D'Études Andines.

Puig, S. 1988: Craneología y craneometría de camélidos: diferenciación interespecífica y determinación de edad. Xama 1: 43-56.

Stanish, CH. 2003: Ancient Titicaca. The evolution of complex society in Southern Peru and Northern Bolivia. University of California Press, Berkeley.

Tomoeda, H. 1993: Los ritos contemporáneos de camélidos y la ceremonia de la citua. En: Millones, L. \& Onuki, Y. (eds.): El mundo ceremonial andino: 289306. Senri Ethnological Studies 37. National Museum of Ethnology, Osaka. 